\title{
Development and validation of a CT radiomics signature for diagnosing COVID-19 pneumonia compared with CO-RADS
}

\section{Huanhuan Liu}

Department of Radiology, Xinhua Hospital, Shanghai Jiao Tong University School of Medicine Hua Ren

Department of Radiology, Xinhua Hospital, Shanghai Jiao Tong University School of Medicine

\section{Zengbin Wu}

Department of Emergency, Xinhua Hospital, Shanghai Jiao Tong University School of Medicine

\section{$\mathrm{He} \mathrm{Xu}$}

Department of Radiology, The First Affiliated Hospital of Bengbu Medical College

\section{Shuhai Zhang}

Department of Radiology, The First Affiliated Hospital of Bengbu Medical College

\section{Jinning Li}

Department of Radiology, Xinhua Hospital, Shanghai Jiao Tong University School of Medicine

\section{Liang Hou}

Department of Radiology, Xinhua Hospital, Shanghai Jiao Tong University School of Medicine

\section{Runmin Chi}

Department of Radiology, Xinhua Hospital, Shanghai Jiao Tong University School of Medicine Hui Zheng

Department of Radiology, Xinhua Hospital, Shanghai Jiao Tong University School of Medicine Yanhong Chen

Department of Radiology, Xinhua Hospital, Shanghai Jiao Tong University School of Medicine Shaofeng Duan

GE Healthcare

\section{Huimin Li}

Department of Radiology, Xinhua Hospital, Shanghai Jiao Tong University School of Medicine

\section{Zongyu Xie}

Department of Radiology, The First Affiliated Hospital of Bengbu Medical College

Dengbin Wang ( $\nabla$ wangdengbin@xinhuamed.com.cn )

Department of Radiology, Xinhua Hospital, Shanghai Jiao Tong University School of Medicine 
Keywords: COVID-19, Computed Tomography, Pneumonia, Radiomics, Machine learning

Posted Date: July 14th, 2020

DOI: https://doi.org/10.21203/rs.3.rs-40940/v1

License: (c) (1) This work is licensed under a Creative Commons Attribution 4.0 International License. Read Full License 


\section{Abstract}

Objectives: To develop and validate a CT radiomics signature for diagnosing COVID-19 pneumonia compared with clinical model and COVID-19 reporting and data system (CO-RADS).

Methods: This two-center retrospective study enrolled 115 laboratory-confirmed COVID-19 patients with 1127 lesions and 435 non-COVID-19 pneumonia patients with 842 lesions. In study 1, a radiomics signature and a clinical model was developed and validated in the training and internal validation cohorts (patient/lesion [n] = 379/1325, $n=131 / 505$ ) for identifying COVID-19 pneumonia. In study 2 , the developed radiomics signature was tested in another independent cohort including all viral pneumonia (n = 40/139), compared with clinical model and CO-RADS approach. The predictive performance was assessed by receiver operating characteristics curve (ROC) analysis, calibration curve, and decision curve analysis (DCA).

Results: Twenty-three texture features were selected to construct the radiomics model. Radiomics model outperformed the clinical model in diagnosing COVID-19 pneumonia with an area under the ROC (AUC) of 0.98 and good calibration in the internal validation cohort. Radiomics model also performed better in the testing cohort to distinguish COVID-19 from other viral pneumonia with an AUC of 0.96 compared with $0.75(P=0.007)$ for clinical model, and $0.69(P=0.002)$ or $0.82(P=0.04)$ for two trained radiologists using CO-RADS approach. The sensitivity and specificity of radiomics model can be improved to 0.90 and 1.00 . The DCA confirmed the clinical utility of radiomics model.

Conclusions: The proposed radiomics signature outperformed clinical model and CO-RADS approach for diagnosing COVID-19, which can facilitate rapid and accurate detection of COVID-19 pneumonia.

\section{Introduction}

The ongoing pandemic of coronavirus disease 2019 (COVID-19) caused by "severe acute respiratory syndrome coronavirus 2" (SARS-CoV-2) has become a global threat [1, 2]. The high contagion of SARSCoV-2 and the virulence to cause severe illness involving multiple organs has caused many countries into a dilemma for screening, diagnosing, and treatment with limited healthcare resources. As of June 20, a total of 8,663,270 worldwide confirmed cases and 460,012 deaths have been reported [3], and the numbers continue to grow. The reverse-transcription polymerase chain reaction (RT-PCR) for SARS-CoV-2 was regarded as the diagnostic gold standard but with various sensitivities ranging from $59 \%$ to $71 \%$ depending on viral load and test sample quality $[4,5]$. Furthermore, the lengthy turnaround time and shortage of RT-PCR kit will delay the treatment, which contributes to the dilemma.

Chest CT imaging is a widely available, time-saving, and non-invasive approach for detecting COVID-19 pneumonia. Previous studies revealed that chest CT could serve as an efficient tool for diagnosing COVID-19 pneumonia with high sensitivity and monitoring disease course [4, 6-8]. Recently, a multinational consensus statement from the Fleischner Society also declared that CT scanning can be a major method if symptoms worsen or there is a situation short of RT-PCR kit [9]. However, COVID-19 
pneumonia shared similar imaging features with pneumonia caused by other pathogens, especially other viral pneumonia. The specificity was relatively low when compared to RT-PCR results [4]. Quarantine for those with final COVID-19 negative RT-PCR results increased stress on limited healthcare resources. As for distinguishing COVID-19 from other viral pneumonia on chest CT, high specificities but moderate sensitivities were reported among different international radiologists [10]. To facilitate the evaluation of COVID-19 pneumonia, a standardized assessment scheme for pulmonary involvement of COVID-19 named CO-RADS (COVID-19 reporting and data system) was developed to estimate the risk [11, 12]. The subjective CO-RADS classification demonstrated high discriminatory power but moderate to substantial agreement among observers. Hence, more measures should be taken for more rapid and accurate diagnosis of COVID-19 to combat the current pandemic.

Radiomics involved high-throughput extraction of a large number of quantitative features from medical images, thereby converting image data into high-dimensional data to objectively and quantitatively describe the characteristics of lesions that may not be perceptible by the naked eye. The potential benefits of radiomics had been highlighted in improving diagnostic, prognostic, and predictive accuracy for cancers such as lung cancer, rectal cancer, etc. as well as other non-neoplastic diseases [13-16]. To date, there are limited data about the value of chest CT-based radiomics in detecting COVID-19 pneumonia.

In the present study, we aimed to develop and validate a radiomics signature model for distinguishing COVID-19 from pneumonia with other etiologies by using real-world data during the COVID-19 outbreak in China. Additionally, the predictive performance of radiomics model was tested in another independent viral pneumonia cohort in comparison with the clinical model and CO-RADS grading approach.

\section{Materials And Methods}

\section{Patients}

This study was approved by the Institutional Ethics Committee of the two enrolled centers. The informed consent requirement was waived for this retrospective study. The workflow of this study was displayed in Figure 1. In study 1, clinical and CT data of consecutive 115 patients with laboratory-confirmed COVID-19 from Bengbu City (center I) as well as 1205 patients with respiratory symptoms from Xinhua Hospital affiliated to Shanghai Jiao Tong University School of Medicine (center II) were reviewed. Patients with common pathogen confirmation and disease improvement on follow-up CT after treatment were grouped as non-COVID-19 pneumonia patients. The exclusion criteria were showed in Figure 1. Consequently, 95 COVID-19 and 415 non-COVID-19 pneumonia patients were recruited, who were semi-randomly allocated to the training and internal validation cohorts according to the recruitment time. In study 2 , another 40 patients with viral pneumonia who met the inclusion and exclusion criteria as an independent cohort were included to test the constructed models. Finally, 115 COVID-19 and 435 non-COVID-19 pneumonia patients were enrolled in this study. Among the non-COVID-19 patients, 128 were confirmed viral 
infections, 195 mycoplasma infections, 5 chlamydia infections, 3 fungus infections, and 104 coinfections.

\section{CT imaging acquisition and interpretation}

All the patients underwent non-enhanced chest CT examinations for detecting pneumonia with a 64section multi-detector CT scanner. The detailed imaging parameters were demonstrated in Appendix E1.

Initial CT images before any treatment were performed by three experienced radiologists in consensus with more than 9 years of experience in thoracic imaging. The disputes among observers were resolved by consulting another experienced radiologist with more than 20 years of experience in thoracic imaging. All of them were blinded to laboratory results.

The lesion number, distribution, density, extent, and other features were assessed. Other features consisted of reticulation, air bronchogram, lymphadenopathy within the mediastinum or hilus, and pleural effusion. Lymphadenopathy was defined as the size of lymph node more than $10 \mathrm{~mm}$ in short-axis diameter. The extent of pulmonary involvement was estimated using a semi-quantitative scoring system. Each of the 5 lung lobe involvements was scored from 0 to 5 as follows: $0(0 \%), 1(<5 \%), 2(5 \%-25 \%), 3$ $(26 \%-49 \%), 4(50 \%-75 \%)$, and 5 (> 75\%) [17].

\section{Image segmentation and radiomics feature extraction}

Three-dimensional (3D) segmentation of the entire volume of interest (VOI) of lesions was performed manually and independently by two experienced radiologists via itk-SNAP 3.8.0 (www.itksnap.org). The outline of lesions was delineated along the border on thick-section images with lung window and excluded intralesional vessels, bronchi, necrosis, and cavitation (Figure 2). The interobserver and intraobserver reproducibility evaluation of radiomics feature extraction was performed using intraclass correlation coefficients (ICC). An ICC of 0.81 to 1.00 showed almost perfect agreement, 0.61 to 0.80 as substantial agreement, and 0.41 to 0.60 as moderate agreement [14].

Radiomics features were extracted from VOls by using pyradiomics 3.0.0 version [18] (http://www.radiomics.io/pyradiomics.html). Six classes of radiomics features were extracted. In addition, two image filters of wavelet and Laplacian of Gaussian were applied to the original image, respectively. Finally, 14 different image types were used for extracting radiomics features. The detailed image segmentation and radiomics feature extraction information was demonstrated in Appendix E2.

\section{Development of clinical and radiomics models}

For clinical model, univariate and multivariate logistic regression analysis were applied to select the independent predictors of clinical and radiological features for diagnosing COVID-19 pneumonia in the training cohort. Then, a clinical nomogram was developed with the selected variables. 
For radiomics signature model, minimum redundancy and maximum relevance (mRMR), and the least absolute shrinkage and selection operator (LASSO) logistic regression were used to select the best performed radiomics features in the training cohort. Radscore was calculated for each lesion and the mean Radscore (mRadscore) of multiple lesions for each patient was used for predicting COVID-19 pneumonia.

\section{Internal validation and clinical utility of clinical and radiomics models}

The predictive performance of clinical and radiomics models was assessed by using the receiver operating characteristic curve (ROC) analysis, in which the areas under the curve (AUCs), accuracies, sensitivities, and specificities were established. Then, the predictive performance of the models was further tested in the internal validation cohort. Calibration curves were performed to assess the goodnessof-fit of the clinical and radiomics models.

Decision curve analysis (DCA) was implemented to evaluate the net benefits of the prediction models at different threshold probabilities in the validation cohort.

\section{Predictive performance of radiomics model in distinguishing COVID-19 from other viral pneumonia compared with clinical model and CO-RADS}

Another independent cohort including 20 COVID-19 patients and 20 patients with other viral pneumonia was used to test the discriminatory power for the clinical model, radiomics model, and CO-RADS. The CORADS included 6 levels of suspicion for pulmonary involvement of COVID-19 besides CO-RADS 0, not interpretable (scan technically insufficient for assigning a score) [11]. The detailed information for each level was demonstrated in Appendix E3.

The CO-RADS categories for the 40 patients were independently performed by two experienced radiologists who were familiar with CO-RADS and blinded to laboratory results. The interobserver agreement was assessed by using Cohen kappa test, where 0.41-0.60 moderate agreement, 0.61-0.80 substantial agreement, and 0.81-1.00 almost perfect agreement [19].

\section{Statistical analysis}

Comparisons of patient characteristics between COVID-19 and non- COVID-19 pneumonia groups were performed by independent two-sample $t$ test, Mann-Whitney $U$ test, and chi-squared test or Fisher's exact test via SPSS 23.0 (IBM). Other statistical analyses were performed with R software (version 3.6.1, http://www.Rproject.org). The AUCs were compared by DeLong test [20]. A two-sided $P<0.05$ indicated a statistically significant difference.

\section{Results}

\section{Patient characteristics}


The clinical and radiological features of the 550 patients in the training, internal validation, and testing cohorts were depicted in the Table 1 and 2. For clinical features, there were significant differences for age, cough symptom, white blood cell count, neutrophil ratio, and lymphocyte count in the training and internal validation cohorts. While in the testing cohort, only C-reactive protein showed significant difference. For radiological features, lesion location and distribution were significantly different between the COVID-19 and non-COVID-19 groups for all the cohorts.

\section{Features selection and development of clinical and radiomics models}

Eight clinical-radiological features were selected for building the clinical model in the training dataset, including age, gender, neutrophil ratio, lymphocyte count, location, distribution, reticulation, and CT score. The developed clinical nomogram was showed in Figure 3.

For radiomics model, 1218 radiomics features were extracted for 783 lesions in 66 COVID-19 patients and 542 lesions in 313 non-COVID-19 patients in the training dataset. The interobserver and intraobserver reproducibility of radiomics feature extraction was satisfactory with ICCs ranging from 0.7139 to 0.9999 , and 0.7130 to 0.9999 , respectively. Twenty-three wavelet-transformed texture features with nonzero coefficients were selected to construct the radiomics signature model (Appendix E4 and Figure S1). The developed radiomics signature was presented in Appendix E5. Radscore of each lesion was depicted in Figure S2.

\section{Validation and clinical utility of clinical and radiomics models}

The AUC of radiomics model developed in the training cohort was 1.00. Favorable performance was observed in the internal validation cohort. The radiomics model outperformed clinical model in diagnosing COVID-19 pneumonia, with an AUC of 0.98 compared with 0.83 . The sensitivity and specificity of radiomics model was improved to 0.91 and 0.94 . The AUCs, accuracies, sensitivities, and specificities of clinical and radiomics models in the training and internal validation cohorts were depicted in Table 3. The ROC analysis results are displayed in Figure 4.

Calibration curves showed that radiomics model demonstrated a better agreement between the predicted and actual probabilities of COVID-19 both in both datasets (Figure S3). DCA revealed that the radiomics prediction model was more beneficial than the clinical model, as well as the "treat-all-patients" or "treatnone" strategies when the threshold probability was from 0.0 to 1.0 (Figure 5).

\section{Predictive performance of clinical model, radiomics model, and CO-RADS category in distinguishing COVID-19 from other viral pneumonia}

In the testing cohort, radiomics model outperformed clinical model in distinguishing COVID-19 from other viral pneumonia with an AUC of 0.96 compared with $0.75(P=0.02)$ (Figure 6). In addition, the radiomics model also performed better than two trained radiologists by using CO-RADS. The AUC of radiomics model was significantly higher than 0.69 for radiologist $1(P=0.002)$ and 0.82 for radiologist $2(P=0.04)$ (Figure 6). The AUCs, accuracies, sensitivities, and specificities of clinical model, radiomics model, and 
CO-RADS in the testing cohort were demonstrated in Table 4. The interobserver agreement between the two radiologists was moderate with a kappa value of 0.53 .

\section{Discussion}

In this study, we developed and validated a CT-based radiomics model for diagnosing COVID-19 pneumonia, and compared its predictive performance with clinical model as well as the performances of two trained radiologists by applying CO-RADS. Our results revealed that radiomics model outperformed clinical model in identifying COVID-19 pneumonia in the training, internal validation, and testing cohorts, and not only for the common pathogens' infections but also for the selective viral infections. The proposed radiomics model achieved favorable performances with AUC values of $1.00,0.98$, and 0.96 as well as high sensitivities and specificities in the three cohorts. Furthermore, the developed radiomics model was also superior to CO-RADS in discriminating COVID-19 from other viral pneumonia with a sensitivity and specificity of 0.90 and 1.00 .

Rapid and accurate diagnosis of COVID-19 is crucial for early intervention and healthcare allocation during the ongoing outbreak. Previous studies had explored the clinical and imaging features of COVID19 for facilitating diagnosis of COVID-19 pneumonia, revealing that fever and/or cough, normal or decreased white blood cells, and decreased lymphocyte count, GGO lesions in the peripheral and posterior lungs on CT images could aid in screening the highly suspicious patients [6, 21-23]. However, more common consolidation lesions could be detected due to the time interval from symptom onset and atypical features including fibrous stripes and irregular solid nodules were also presented in the subsequent studies, which complicated the diagnosis [8,24]. Our study found that older age, male, normal neutrophil ratio, decrease lymphocyte count, bilateral locations, peripheral distributions, and reticulation on CT as well as CT score were independent predictors for distinguishing COVID-19 from nonCOVID-19 pneumonia derived from the training cohort, which was in accordance with the above studies. Nevertheless, the predictive performance was not satisfactory with an AUC of 0.83 and a sensitivity of 0.63 in the internal validation dataset. The various sensitivities and specificities of identifying COVID-19 subjectively with the clinical and radiological features were also found in previous studies $[4,5,10]$.

When evaluating the diagnostic performance of clinical model in discriminating COVID-19 from other viral pneumonia in the testing dataset, the discriminatory power further decreased with an AUC and sensitivity of 0.75 and 0.60 . In prior investigations conducting comparison between chest CT and RT-PCR results, the sensitivity of CT in identifying COVID-19 pneumonia can be estimated to $98 \%$, but the specificity was only $25 \%$ by analyzing 1014 patients $[4,5]$. Regarding the diagnostic performance among different radiologists from different countries in distinguishing COVID-19 from viral pneumonia on chest $\mathrm{CT}$, the sensitivity, however, was reported to be moderate but the specificity was high [10]. Even by applying the recently recommended CO-RADS with reported high discriminatory power of AUC 0.91 in identifying COVID-19 [11], the AUC, sensitivity, and specificity in our study were not satisfactory with 0.69 , 0.80 , and 0.55 , respectively for a trained radiologist who was familiar with CO-RADS, as well as $0.82,0.9$, and 0.65 , respectively for another trained radiologist in distinguishing COVID-19 from other viral 
pneumonia. The moderate inter-observer agreement was also not in favor of the accurate diagnosis of COVID-19. Therefore, it is urgent to develop a more objective approach for improving the current diagnostic accuracy of COVID-19 pneumonia.

Recently, artificial intelligence (Al) using deep learning technology has demonstrated good performance to improve the diagnosis of COVID-19, with sensitivities ranging from 0.67 to 0.90 and specificities from 0.83 to 0.96 [25-27]. However, deep learning requires a large amount of data to be trained, which limited its timely application based on the sporadic COVID-19 cases in most parts of China. More clinical implementations are warranted for the test of Al system and wide availability. Another machine learning approach radiomics rapidly developed in recently years can be widely available through open-source software and the radiomics signature is easily utilized. The potential for diagnosing and predicting outcomes of different lesions has been proven in the prior reproducible investigations $[14,15]$, as well as our previous studies in predicting preoperative synchronous distant metastasis in patients with rectal cancer $[28,29]$. In this study, 23 textural features were selected to build the radiomics model and the proposed model performed well not only in the training cohort but also in the two validation cohort with AUCs of $1.00,0.98$, and 0.96 , respectively. The high sensitivities and specificities with 1.00 and 0.97 in the training cohort as well as 0.91 and 0.94 in the internal validation cohort were observed.

It was reported that there were overlaps in imaging findings between COVID-19 and other viral infections, such as the coronavirus SARS-CoV and MERS-CoV pneumonia, as well as H1N1, H5N1, influenza, and so on $[6,23,30]$. Therefore, it is not difficult to understand that the textural features outperformed the other extracted morphological features or the first-order statistical features according to the histogram analysis. Textural features encoded the relationships between nearby voxels within VOls, reflecting the intralesional heterogeneity. It is the advantage that radiomics can transform conventional medical images into quantitative and high-dimensional data visual analysis [31, 32]. To further test the robustness of our developed radiomics model, we enrolled an independent validation cohort including viral infection patients to assess the diagnostic performance. The AUC, accuracy, sensitivity, and specificity were satisfactory with values of $0.96,0.95,0.90$, and 1.00 , respectively. When compared with the clinical model and the CO-RADS for identifying COVID-19 pneumonia, the AUC value of radiomics model was significantly higher. The high sensitivity and specificity can not only facilitate to select the highly suspicious patients of COVID-19 for timely management, but also help to exclude the negative patients for relieving the stress of healthcare system.

Our study has several limitations. First, the sample size of patients in our retrospective study was relatively small. Prospective investigation with a larger sample size from more centers will be required to validate our proposed model. Second, since we enrolled the non-COVID pneumonia patients with blood laboratory pathogen-confirmation and pneumonia improvement after treatment by follow-up CT scans, limited bacterial infection cases were available due to the lack of bacterial culture. Third, center II were a general hospital with a strong pediatric medical center, thus many children with mycoplasma infections were included in our study. The median age was demonstrated significantly lower than that of the COVID19 infection patients, where selection bias may exist. However, our non-COVID-19 pneumonia cases were 
consecutively enrolled from the real word data in our center, and the children was also proved to be susceptible for COVID-19, which definitely needed rapid and accurate differential diagnosis.

In summary, our preliminary study demonstrated that chest CT-based radiomics signature model outperformed clinical model and CO-RADS in diagnosing COVID-19 pneumonia. The useful quantitative lesion characteristics derived from the proposed radiomics model can facilitate rapid and accurate diagnosis as well as timely management of COVID-19.

\section{Declarations}

\section{Funding}

This work was supported by Special Research Program of Shanghai Municipal Commission of Heath and Family Planning on medical intelligence [No. 2018ZHYL0108], National Key Research and Development Program of China [No. 2017YFC0109003], National Natural Science Foundation of China [No. 81901695], and Shanghai Sailing Program [No.19YF1433100]. The funders had no role in study design, data collection and analysis, decision to publish, or preparation of the manuscript.

\section{Conflict of interest}

One of the authors (SD) is an employee of GE Healthcare. The remaining authors of this manuscript declare no relationships with any companies whose products or services may be related to the subject matter of the article.

\section{References}

$1 \quad$ World Health Organization. WHO Director-General's remarks at the media briefing on 2019-nCoV on 11 February 2020. 2020. at https://www.who.int/dg/speeches/detail/who-director-general-s-remarks-atthe-media-briefing-on-2019-ncov-on-11-february-2020. Accessed February 11, 2020

2 World Health Organization. WHO Director-General's opening remarks at the media briefing on COVID-19 - 11 March 2020. at https://www.who.int/dg/speeches/detail/who-director-general-s-openingremarks-at-the-media-briefing-on-covid-19-11-march-2020, Accessed March 11, 2020

3 Johns Hopkinds University \& Medicine, C.R.C., (2020). https://coronavirus.jhu.edu/ map.html (accessed June 20, 2020)

$4 \quad \mathrm{Ai}$ T, Yang Z, Hou H et al (2020) Correlation of Chest CT and RT-PCR Testing in Coronavirus Disease 2019 (COVID-19) in China: A Report of 1014 Cases. Radiology, 200642

5 Fang Y, Zhang H, Xie J et al (2020) Sensitivity of Chest CT for COVID-19: Comparison to RT-PCR. Radiology, 200432 
6 Song F, Shi N, Shan F et al (2020) Emerging Coronavirus 2019-nCoV Pneumonia. Radiology 295, $210-217$

7 Shi $\mathrm{H}, \mathrm{Han} \mathrm{X}$, Jiang $\mathrm{N}$ et al Radiological findings from 81 patients with COVID-19 pneumonia in Wuhan, China: a descriptive study. The Lancet Infectious Diseases 20,425-434

8 Pan F, Ye T, Sun P et al (2020) Time Course of Lung Changes On Chest CT During Recovery From 2019 Novel Coronavirus (COVID-19) Pneumonia. Radiology 295, 715-721

9 Rubin GD, Ryerson CJ, Haramati LB et al (2020) The Role of Chest Imaging in Patient Management during the COVID-19 Pandemic: A Multinational Consensus Statement from the Fleischner Society. Radiology, 201365

10 Bai HX, Hsieh B, Xiong Z et al (2020) Performance of radiologists in differentiating COVID-19 from viral pneumonia on chest CT. Radiology, 200823

11 Prokop M, van Everdingen W, van Rees Vellinga T et al (2020) CO-RADS - A categorical CT assessment scheme for patients with suspected COVID-19: definition and evaluation. Radiology, 201473

12 Salehi S, Abedi A, Balakrishnan S, Gholamrezanezhad A (2020) Coronavirus disease 2019 (COVID$19)$ imaging reporting and data system (COVID-RADS) and common lexicon: a proposal based on the imaging data of 37 studies. Eur Radiol. Doi:10.1007/s00330-020-06863-0. Online ahead of print

13 Guiot J, Vaidyanathan A, Deprez L et al (2020) Development and validation of an automated radiomic CT signature for detecting COVID-19. medRxiv, 2020.2004.2028.20082966

14 Liu Z, Zhang XY, Shi YJ et al (2017) Radiomics Analysis for Evaluation of Pathological Complete Response to Neoadjuvant Chemoradiotherapy in Locally Advanced Rectal Cancer. Clin Cancer Res 23:7253-7262

15 Chen BT, Chen Z, Ye N et al (2020) Differentiating Peripherally-Located Small Cell Lung Cancer From Non-small Cell Lung Cancer Using a CT Radiomic Approach. Front Oncol 10:593

16 Liu Y, Dong D, Zhang L et al (2019) Radiomics in multiple sclerosis and neuromyelitis optica spectrum disorder. Eur Radiol 29:4670-4677

17 Chang YC, Yu CJ, Chang SC et al (2005) Pulmonary sequelae in convalescent patients after severe acute respiratory syndrome: evaluation with thin-section CT. Radiology 236:1067-1075

18 van Griethuysen JJM, Fedorov A, Parmar C et al (2017) Computational Radiomics System to Decode the Radiographic Phenotype. Cancer Res 77:e104-e107

19 Chen X, Feng B, Li C et al (2020) A radiomics model to predict the invasiveness of thymic epithelial tumors based on contrast-enhanced computed tomography. Oncol Rep 43:1256-1266 
20 DeLong ER, DeLong DM, Clarke-Pearson DL (1988) Comparing the areas under two or more correlated receiver operating characteristic curves: a nonparametric approach. Biometrics 44:837-845

21 Wang D, Hu B, Hu C et al (2020) Clinical Characteristics of 138 Hospitalized Patients With 2019 Novel Coronavirus-Infected Pneumonia in Wuhan, China. JAMA 323, 1061-1069

22 Chen N, Zhou M, Dong X et al (2020) Epidemiological and clinical characteristics of 99 cases of 2019 novel coronavirus pneumonia in Wuhan, China: a descriptive study. Lancet 395:507-513

23 Chung M, Bernheim A, Mei X et al (2020) CT Imaging Features of 2019 Novel Coronavirus (2019nCoV). Radiology, 200230

24 Pan Y, Guan H, Zhou S et al (2020) Initial CT findings and temporal changes in patients with the novel coronavirus pneumonia (2019-nCoV): a study of 63 patients in Wuhan, China. Eur Radiol 30,33063309

25 Bai HX, Wang R, Xiong Z et al (2020) Al Augmentation of Radiologist Performance in Distinguishing COVID-19 from Pneumonia of Other Etiology on Chest CT. Radiology, 201491

26 Li L, Qin L, Xu Z et al (2020) Artificial Intelligence Distinguishes COVID-19 from Community Acquired Pneumonia on Chest CT. Radiology, 200905

27 Wang S, Kang B, Ma J et al (2020) A deep learning algorithm using CT images to screen for Corona Virus Disease (COVID-19). medRxiv, 2020.2002.2014.20023028

28 Cui Y, Liu H, Ren J et al (2020) Development and validation of a MRI-based radiomics signature for prediction of KRAS mutation in rectal cancer. Eur Radiol 30:1948-1958

29 Liu H, Zhang C, Wang L et al (2019) MRI radiomics analysis for predicting preoperative synchronous distant metastasis in patients with rectal cancer. Eur Radiol 29:4418-4426

30 Koo HJ, Lim S, Choe J, Choi SH, Sung H, Do KH (2018) Radiographic and CT Features of Viral Pneumonia. Radiographics 38:719-739

31 Gillies RJ, Kinahan PE, Hricak H (2016) Radiomics: Images Are More than Pictures, They Are Data. Radiology 278:563-577

32 Lambin P, Leijenaar RTH, Deist TM et al (2017) Radiomics: the bridge between medical imaging and personalized medicine. Nat Rev Clin Oncol 14:749-762

\section{Tables}

Table 1. Clinical characteristics of patients with COVID-19 and non-COVID-19 pneumonia in the training and validation cohorts 


\begin{tabular}{|c|c|c|c|c|c|c|c|c|c|}
\hline \multirow[t]{2}{*}{ Characteristics } & \multicolumn{2}{|c|}{ Training cohort $(n=379)$} & \multirow[t]{2}{*}{$P$ value } & \multicolumn{2}{|c|}{ Internal validation cohort $(n=131)$} & \multirow[t]{2}{*}{$P$ value } & \multicolumn{2}{|c|}{ Testing cohort $(n=40)$} & \multirow[t]{2}{*}{$P$ value } \\
\hline & $\begin{array}{c}\text { non- } \\
\text { COVID-19 } \\
\text { group }(n=313)\end{array}$ & $\begin{array}{l}\text { COVID-19 group } \\
\qquad(\mathrm{n}=66)\end{array}$ & & $\begin{array}{l}\text { non-COVID-19 } \\
\text { group }(n=102)\end{array}$ & $\begin{array}{l}\text { COVID-19 } \\
\text { group }(n=29)\end{array}$ & & $\begin{array}{l}\text { non-COVID-19 } \\
\text { group }(n=20)\end{array}$ & $\begin{array}{l}\text { COVID-19 } \\
\text { group }(n=20)\end{array}$ & \\
\hline $\begin{array}{l}\text { Age, median } \\
\text { (IQR), years }\end{array}$ & $7.0(26.0)$ & $47.5(20.5)$ & $\begin{array}{c}< \\
0.001\end{array}$ & $30.5(49.2)$ & $40.0(20.5)$ & 0.019 & $56.5(30.5)$ & $43.0(13.0)$ & 0.081 \\
\hline Gender (\%) & & & 0.006 & & & 0.587 & & & 0.749 \\
\hline Female & $177(56.5)$ & $25(37.9)$ & & $62(60.8)$ & $16(55.2)$ & & $9(45.0)$ & $8(40.0)$ & \\
\hline Male & $136(43.5)$ & $41(62.1)$ & & $40(39.2)$ & $13(44.8)$ & & $11(55.0)$ & $12(60.0)$ & \\
\hline \multicolumn{10}{|l|}{$\begin{array}{l}\text { Initial symptoms } \\
(\%)\end{array}$} \\
\hline Snotty & $6(1.9)$ & $3(4.5)$ & 0.407 & $0(0.0)$ & $1(3.4)$ & 0.221 & $0(0.0)$ & $1(5.0)$ & 0.500 \\
\hline Sore throat & $7(2.2)$ & $4(6.1)$ & 0.201 & $9(8.8)$ & $6(20.7)$ & 0.15 & $0(0.0)$ & $2(10.0)$ & 0.468 \\
\hline Sputum & $123(39.3)$ & $20(30.3)$ & 0.171 & $18(17.6)$ & $6(20.7)$ & 0.709 & $2(10.0)$ & $5(25.0)$ & 0.405 \\
\hline Fever & $196(62.6)$ & $48(72.7)$ & 0.119 & $65(63.7)$ & $24(82.8)$ & 0.053 & $11(55.0)$ & $14(70.0)$ & 0.327 \\
\hline Dyspnoea & $3(1.0)$ & $4(6.1)$ & 0.022 & $2(2.0)$ & $1(3.4)$ & 0.531 & $0(0.0)$ & $1(5.0)$ & 0.500 \\
\hline \multicolumn{10}{|l|}{$\begin{array}{l}\text { Laboratory test* } \\
(\%)\end{array}$} \\
\hline $\begin{array}{l}\text { White blood cell } \\
\text { count }\end{array}$ & $\begin{array}{c}127(40.6) / 17 \\
(5.4)\end{array}$ & $\begin{array}{c}9(13.6) / 15 \\
(22.7)\end{array}$ & $\begin{array}{c}< \\
0.001\end{array}$ & $22(21.6) / 5(4.9)$ & $0(0.0) / 6(20.7)$ & 0.014 & $7(35.0) / 0(0.0)$ & $\begin{array}{c}3(15.0) / 3 \\
(15.0)\end{array}$ & 0.098 \\
\hline $\begin{array}{l}\text { Neutrophil } \\
\text { count }\end{array}$ & $\begin{array}{c}70(22.4) / 16 \\
(5.1)\end{array}$ & $\begin{array}{c}10(15.2) / 3 \\
(4.5)\end{array}$ & 0.402 & $28(27.5) / 2(2.0)$ & $8(27.6) / 1(3.4)$ & 0.989 & $8(40.0) / 0(0.0)$ & $5(25.0) / 0(0.0)$ & 0.311 \\
\hline Neutrophil ratio & $\begin{array}{c}114(36.4) / 34 \\
(10.9)\end{array}$ & $9(13.6) / 4(6.1)$ & $\begin{array}{c}< \\
0.001\end{array}$ & $43(42.2) / 12(11.8)$ & $5(17.2) / 2(6.9)$ & 0.017 & $9(45.0) / 0(0.0)$ & $5(25.0) / 0(0.0)$ & 0.185 \\
\hline $\begin{array}{l}\text { Lymphocyte } \\
\text { count }\end{array}$ & $\begin{array}{c}68(21.7) / 74 \\
(23.6)\end{array}$ & $\begin{array}{c}2(3.0) / 36 \\
(54.5)\end{array}$ & $\begin{array}{c}< \\
0.001\end{array}$ & $5(4.9) / 32(31.4)$ & $\begin{array}{c}0(0.0) / 17 \\
(58.6)\end{array}$ & 0.007 & $0(0.0) / 10(50.0)$ & $0(0.0) / 5(25.0)$ & 0.102 \\
\hline $\begin{array}{l}\text { C-reactive } \\
\text { protein }\end{array}$ & $\begin{array}{c}165(52.7) / 0 \\
(0.0)\end{array}$ & $\begin{array}{c}43(65.2) / 0 \\
(0.0)\end{array}$ & 0.065 & $72(70.6) / 0(0.0)$ & $\begin{array}{c}22(75.9) / 0 \\
(0.0)\end{array}$ & 0.578 & $18(90.0) / 0(0.0)$ & $\begin{array}{c}11(55.0) / 0 \\
(0.0)\end{array}$ & 0.013 \\
\hline
\end{tabular}

IQR, inter-quartile range

*Data are shown in the order of elevated and decreased results for the laboratory tests.

Table 2. Radiological characteristics of patients with COVID-19 and non-COVID-19 pneumonia in the training and validation cohorts 


\begin{tabular}{|c|c|c|c|c|c|c|c|c|c|}
\hline \multirow[t]{2}{*}{ Characteristics } & \multicolumn{2}{|c|}{ Training cohort $(n=379)$} & \multirow[t]{2}{*}{$P$ value } & \multicolumn{2}{|c|}{ Internal validation cohort $(n=131)$} & \multirow[t]{2}{*}{$P$ value } & \multicolumn{2}{|c|}{ Testing cohort $(n=40)$} & \multirow{2}{*}{$\begin{array}{l}P \\
\text { value }\end{array}$} \\
\hline & $\begin{array}{l}\text { non-COVID-19 } \\
\text { group }(n=313)\end{array}$ & $\begin{array}{c}\text { COVID-19 } \\
\text { group }(n=66)\end{array}$ & & $\begin{array}{l}\text { non-COVID-19 } \\
\text { group }(n=102)\end{array}$ & $\begin{array}{c}\text { COVID-19 } \\
\text { group }(n=29)\end{array}$ & & $\begin{array}{r}\text { non-COVID-19 } \\
\text { group }(n=20)\end{array}$ & $\begin{array}{c}\text { COVID-19 } \\
\text { group }(n=20)\end{array}$ & \\
\hline Number (\%) & & & $\begin{array}{c}< \\
0.001\end{array}$ & & & 0.001 & & & 0.025 \\
\hline Single & $179(57.2)$ & $5(7.6)$ & & $64(62.7)$ & $8(27.6)$ & & $12(60.0)$ & $5(25.0)$ & \\
\hline Multiple & $134(42.8)$ & $61(92.4)$ & & $38(37.3)$ & $21(72.4)$ & & $8(40.0)$ & $15(75.0)$ & \\
\hline Location (\%) & & & 0.131 & & & $>0.999$ & & & 0.339 \\
\hline Unilateral lung & $33(10.5)$ & $3(4.5)$ & & $6(5.9)$ & $2(6.9)$ & & $4(20.0)$ & $1(5.0)$ & \\
\hline Bilateral lungs & $280(89.5)$ & $63(95.5)$ & & $96(94.1)$ & $27(93.1)$ & & $16(80.0)$ & $19(95.0)$ & \\
\hline $\begin{array}{l}\text { Predominant } \\
\text { distribution (\%) }\end{array}$ & & & $\begin{array}{c}< \\
0.001\end{array}$ & & & $\begin{array}{c}< \\
0.001\end{array}$ & & & 0.006 \\
\hline Central & $24(7.7)$ & $1(1.5)$ & & $3(2.9)$ & $1(3.4)$ & & $1(5.0)$ & $0(0.0)$ & \\
\hline $\begin{array}{l}\text { Peripheral and } \\
\text { Central }\end{array}$ & $241(77.0)$ & $19(28.8)$ & & $80(78.4)$ & $13(44.8)$ & & $13(65.0)$ & $4(20.0)$ & \\
\hline Density (\%) & & & 0.544 & & & 0.469 & & & 0.067 \\
\hline Pure GGO & $76(24.3)$ & $13(19.7)$ & & $20(19.6)$ & $8(27.6)$ & & $11(55.0)$ & $4(20.0)$ & \\
\hline $\begin{array}{l}\text { Pure } \\
\text { consolidation }\end{array}$ & $75(24.0)$ & $14(21.2)$ & & $23(22.5)$ & $4(13.8)$ & & $3(15.0)$ & $4(20.0)$ & \\
\hline $\begin{array}{c}\text { GGO with } \\
\text { consolidation }\end{array}$ & $162(51.8)$ & $39(59.1)$ & & $59(57.8)$ & $17(58.6)$ & & $6(30.0)$ & $12(60.0)$ & \\
\hline \multicolumn{10}{|l|}{$\begin{array}{l}\text { Accompanying } \\
\text { features }(\%)\end{array}$} \\
\hline Reticulation & $22(7.0)$ & $27(40.9)$ & $\begin{array}{c}< \\
0.001\end{array}$ & $13(12.7)$ & $8(27.6)$ & 0.102 & $9(45.0)$ & $7(35.0)$ & 0.519 \\
\hline \multicolumn{10}{|l|}{ Other findings (\%) } \\
\hline Pleural effusion & $31(9.9)$ & $10(15.2)$ & 0.212 & $12(11.8)$ & $2(6.9)$ & 0.683 & $1(5.0)$ & $0(0.0)$ & $\begin{array}{c}> \\
0.999\end{array}$ \\
\hline Lymphadenopathy & $46(14.7)$ & $1(1.5)$ & 0.003 & $11(10.8)$ & $2(6.9)$ & 0.790 & $2(10.0)$ & $0(0.0)$ & 0.468 \\
\hline $\begin{array}{l}\text { CT score, median } \\
\text { (IQR) }\end{array}$ & $4.0(3.0)$ & $5.0(3.0)$ & $\begin{array}{c}< \\
0.001\end{array}$ & $3.0(3.0)$ & $5.0(4.5)$ & 0.042 & $2.5(3.5)$ & $4.5(4.8)$ & 0.157 \\
\hline
\end{tabular}

IQR, inter-quartile range

Table 3. Predictive performance of clinical and radiomics models for diagnosing COVID-19 pneumonia in the training and internal validation cohorts

\begin{tabular}{ccccc}
\hline & \multicolumn{2}{c}{ Training cohort } & \multicolumn{2}{c}{ Validation cohort } \\
\cline { 2 - 5 } & Clinical model & Radiomics model & Clinical model & Radiomics model \\
\hline AUC (95\% CI) & $0.95(0.93-0.98)$ & $1.00(0.93-0.98)$ & $0.83(0.75-0.90)$ & $0.98(0.95-1.00)$ \\
\hline Accuracy & 0.92 & 0.97 & 0.79 & 0.93 \\
Sensitivity & 0.89 & 1.00 & 0.63 & 0.91 \\
Specificity & 0.93 & 0.97 & 0.84 & 0.94 \\
\hline
\end{tabular}

AUC, area under the receiver operating characteristic curve; CI, confidence interval

Table 4. Predictive performance of clinical model, radiomics model, and CO-RADS in distinguishing COVID-19 pneumonia from other viral pneumonia in the testing cohort 


\begin{tabular}{ccccc}
\hline & Clinical model & Radiomics model & \multicolumn{2}{c}{ CO-RADS } \\
\cline { 3 - 5 } & & & Radiologist 1 & Radiologist 2 \\
\hline AUC (95\% CI) & $0.75(0.60-0.90)$ & $0.96(0.90-1.00)$ & $0.69(0.53-0.85)$ & $0.82(0.70-0.95)$ \\
\hline Accuracy & 0.65 & 0.95 & 0.68 & 0.78 \\
\hline Sensitivity & 0.60 & 0.90 & 0.80 & 0.90 \\
\hline Specificity & 0.70 & 1.00 & 0.55 & 0.65 \\
\hline
\end{tabular}

AUC, area under the receiver operating characteristic curve; CI, confidence interval

CO-RADS, COVID-19 reporting and data system

\section{Figures}

Study 1: To develop and internally validate a radiomics signature model for distinguishing COVID-19 from non-COVID-19 pneumonia compared with clinical model

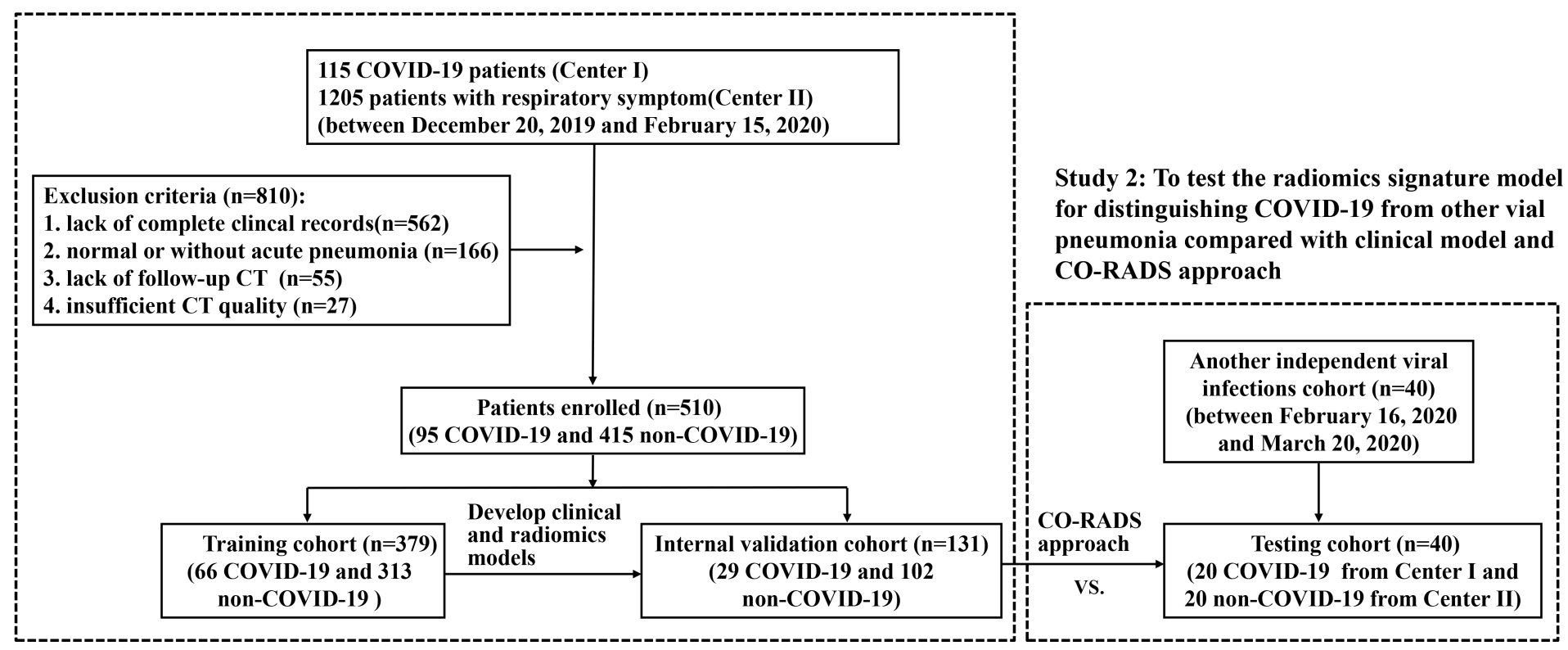

\section{Figure 1}

The workflow of this study 


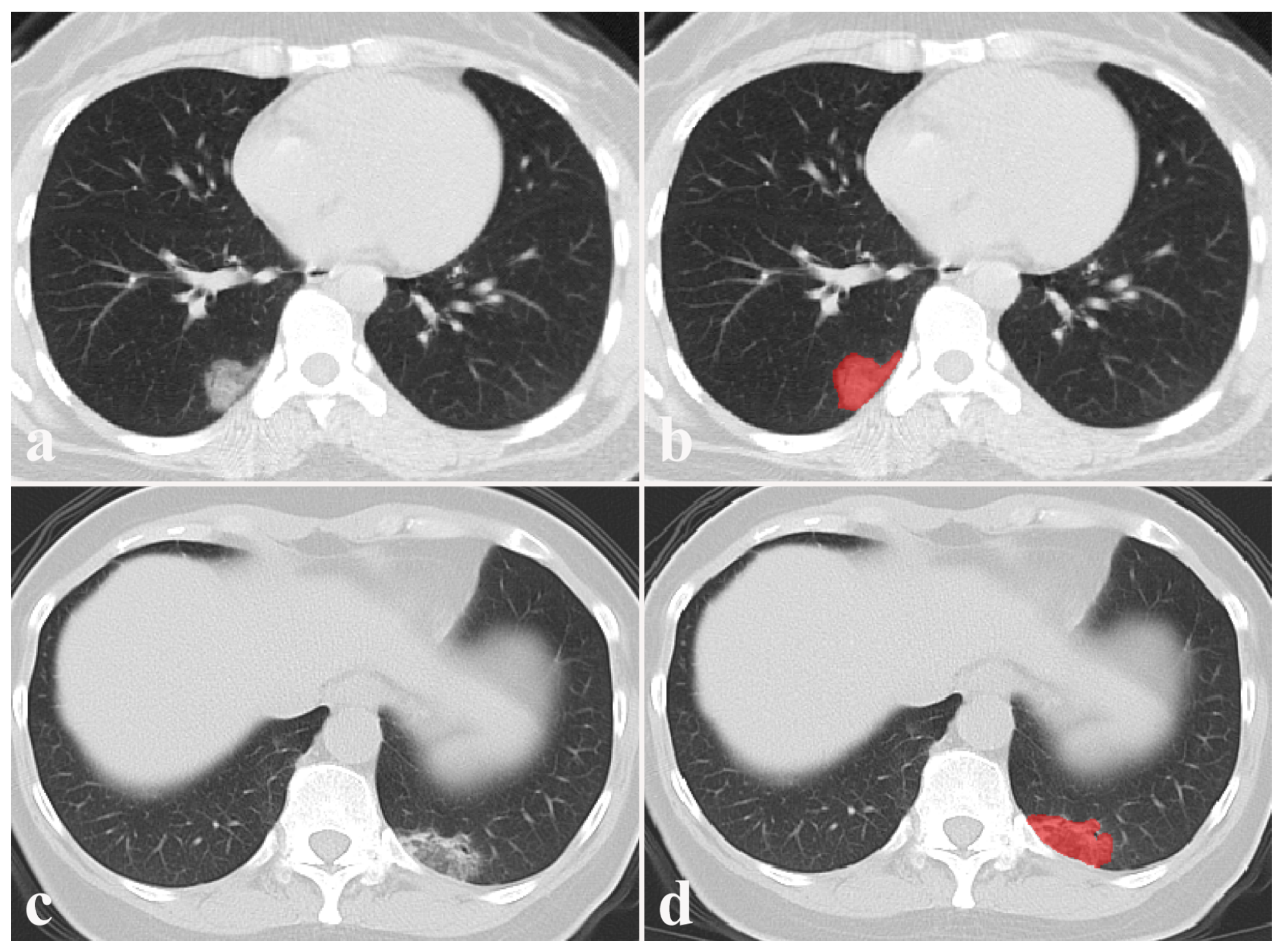

Figure 2

Manual segmentation of COVID-19 pneumonia (2a, 2b) and non- COVID-19 pneumonia lesions (2c, 2d) 
Points

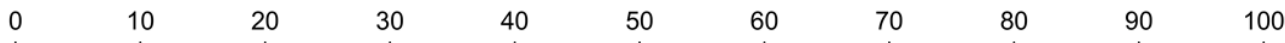

Age

Distribution

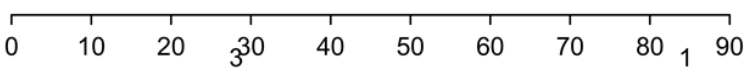

CTscore

Neutrophils_ratio

Lymphocyte Count

Gender

Location

Reticulation

Total Points

Risk

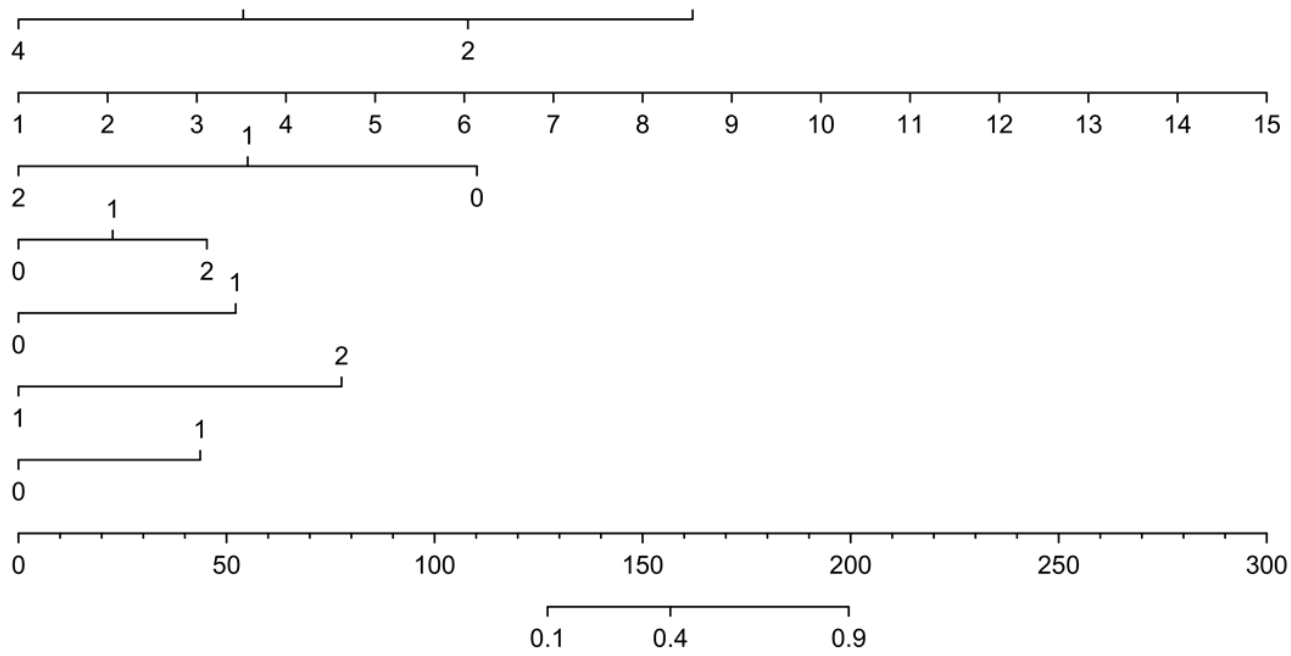

\section{Figure 3}

The developed clinical nomogram for predicting COVID-19 pneumonia in the training cohort, including 4 clinical features and 4 radiological features
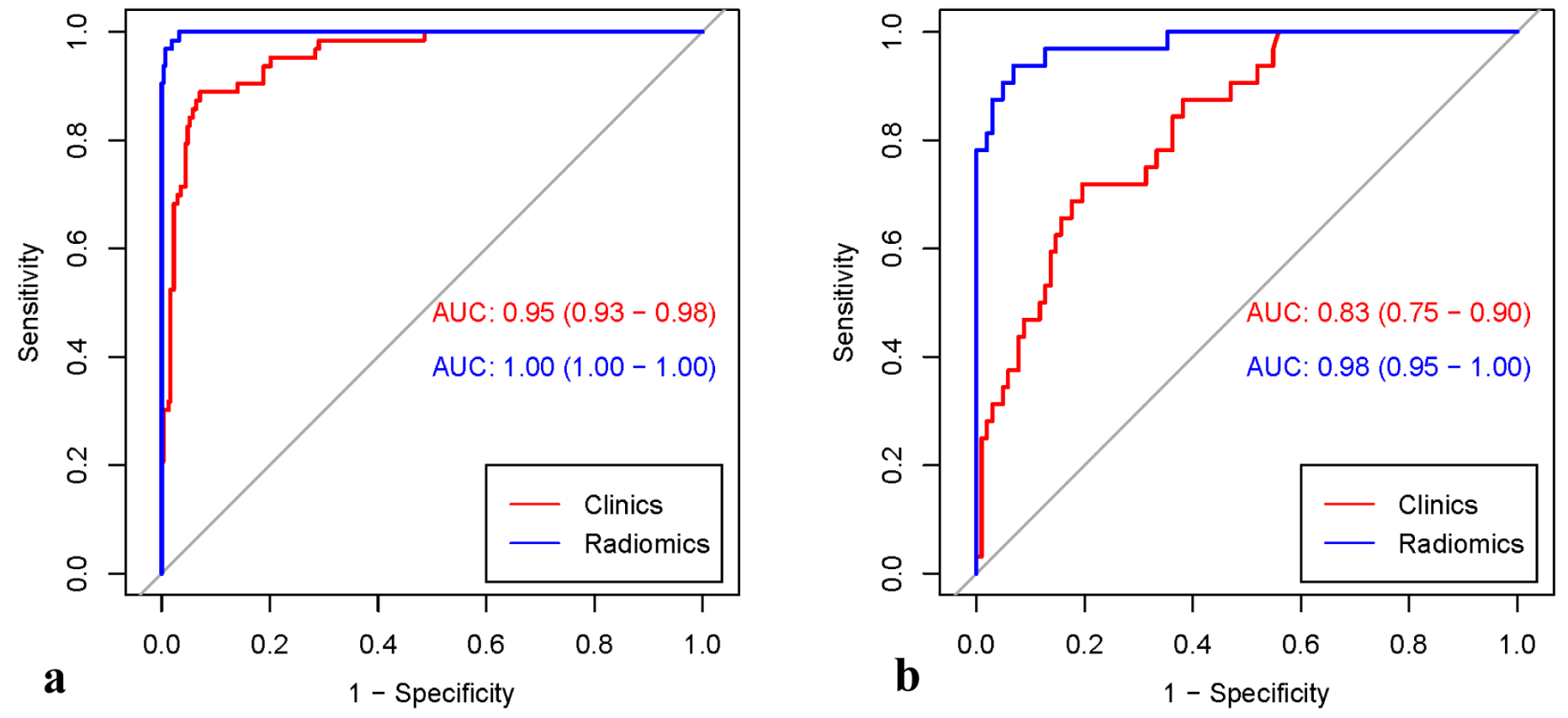

Figure 4

Receiver operating characteristic curve (ROC) analysis for the clinical model and radiomics model in the training cohort (a) and internal validation cohort (b). The diagnostic performance of the radiomics model 
in distinguishing COVID-19 from pneumonia with other pathogens was better than that of the clinical model in both of training and validation cohorts.

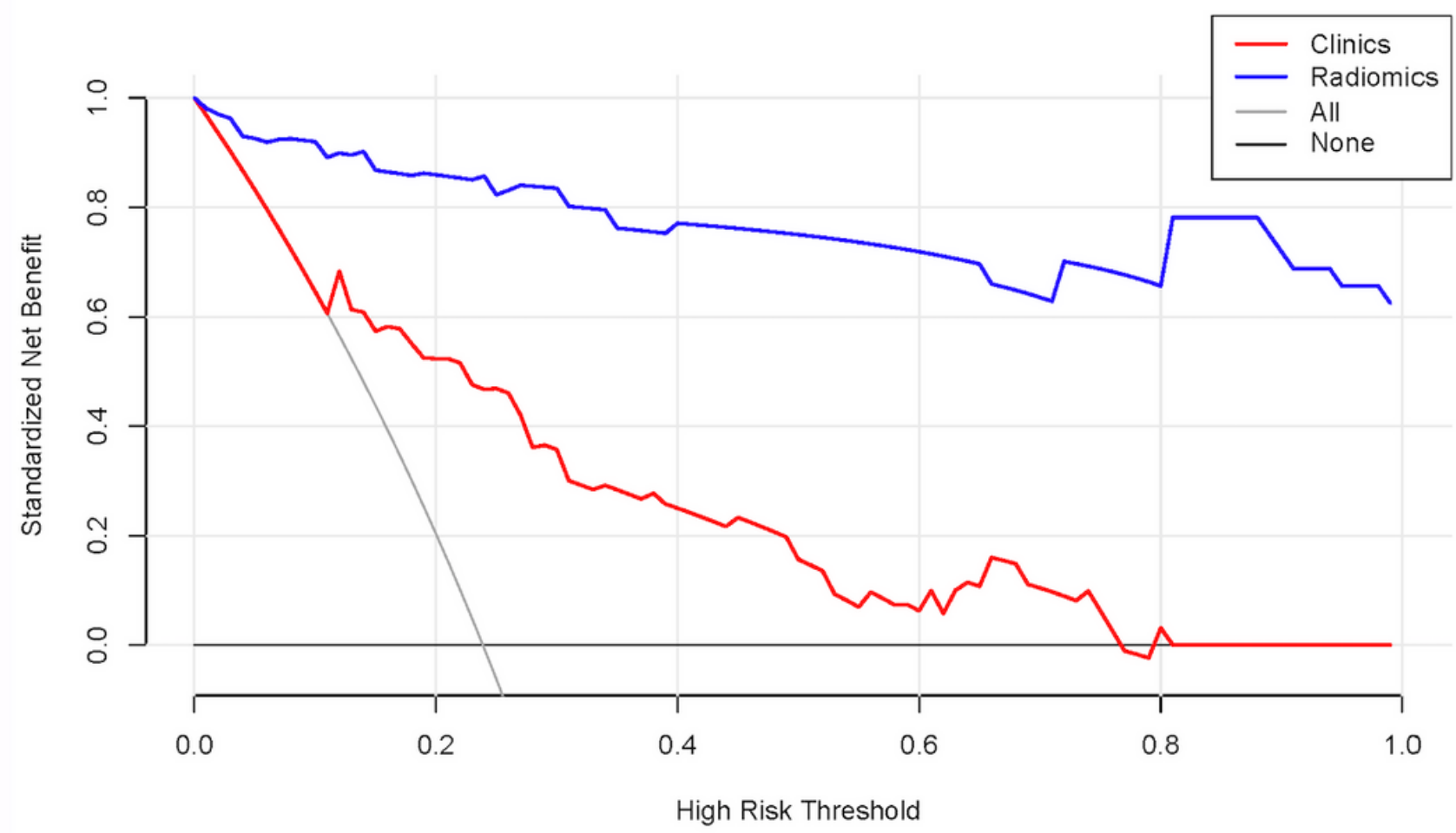

Figure 5

Decision curve analysis for the clinical model and radiomics. The decision curve showed that a radiomics combined model to predict COVID-19 would be more beneficial than the clinical model when the threshold probability was from 0.0 to 1.0 . 


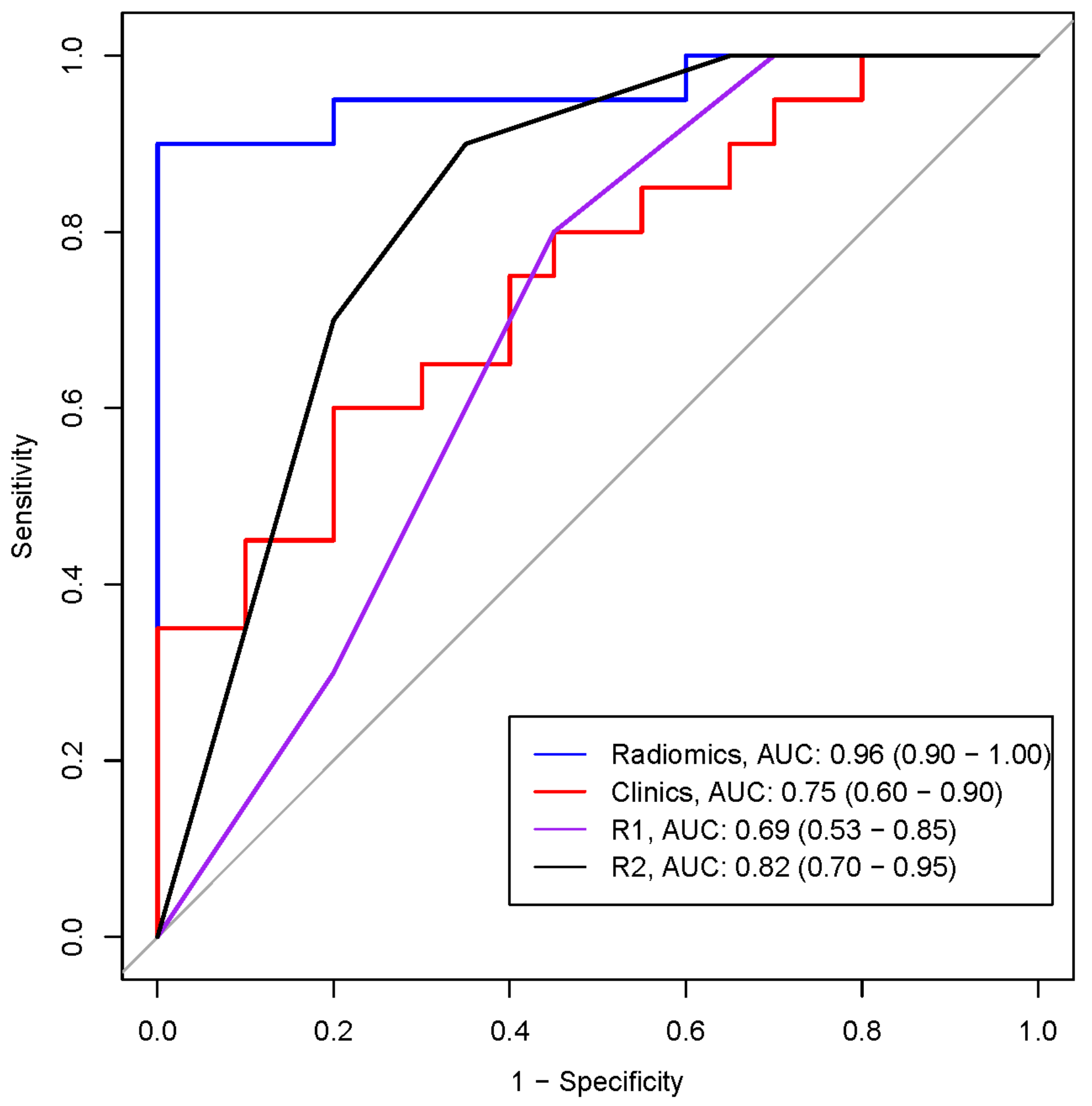

Figure 6

ROC analysis for the clinical model, radiomics model, and CO-RADS approach in the external validation cohort. The diagnostic performance of the radiomics model in distinguishing COVID-19 from pneumonia with other viral pneumonia was better than those of the clinical model and CO-RADS approach.

\section{Supplementary Files}


This is a list of supplementary files associated with this preprint. Click to download.

- Supplementalmaterial.docx

- FigureS1.tif

- Figures2.tif

- FigureS3.tif 